\title{
Design of a Permanent Magnet Electron-Cyclotron Resonance (ECR) Ion Source
}

\author{
Wayne D. Cornelius, Scientific Solutions, PO Box 500207, San Diego CA 92150
}

\section{Abstract}

A design for a permanent magnet electron cyclotron resonance (PM-ECR) ion source is presented. This PMECR ion source was originally designed to substitute for the electromagnet ECR source in the LEDA injector system at Los Alamos National Laboratory and was designed to duplicate, as much as was practical, the parameters of that ion source as detailed in recent publications. To maximize the utility of this ion source for producing other positive ion beams, this particular design is very flexible. The plasma chamber, the beam formation aperture, and the rf feed system are fieldreplaceable to facilitate the specific requirements of different ion beams. The design of the permanent magnet solenoid is quite flexible as well. The baseline magnetic field profile is set during fabrication by adjusting permanent magnet strengths to optimize the field shape for a particular range of ion species. Further, the magnetic field can be adjusted on-line (within certain limits) to optimize the performance of the ion source for a particular ion species. The permanent magnet eliminates all requirements for active components at high voltage. The only connections that cross the high-voltage boundary are the rf waveguide and the source gas feed lines.

\section{PMECR SOURCE DESIGN FEATURES}

The design of the PM-ECR ion source is quite flexible. The next two sections discuss the standard features of the baseline ion source. The following section illustrates the flexibility of the design by discussing various other features, additions, and modifications that are supported by this design.

\subsection{Magnetic Field Profile Design}

The solenoidal magnetic field of the PM-ECR ion source is produced by permanent magnets rather than electromagnets. The important differences between conventional electromagnet ECR sources and this permanent magnet (PM) version are 1) the absence of a high-current power supply, magnetic coils, and associated cooling system and 2) the potential to significantly reduce magnetic field intensity at the beam formation aperture.

Stokes Law requires that the integral of magnetic field around any closed contour be equal to the current enclosed within that contour. Since a permanent magnet solenoid contains no current, such an integral must be identically zero. Hence the magnetic field must change sign near both ends of a permanent magnet solenoid such that the integral of the magnetic field external to the solenoid precisely cancels the integral of the internal field. Figure 1 compares the magnetic field of a PM solenoid with the field of an electromagnet solenoid.[1] Note the field reversal near the plasma aperture in the right-hand side of the figure. This field-reversal feature allows us to locate the beam formation electrode in the low-field regions, possibly resulting in reduced beam emittance compared with electro-magnet ECR sources.

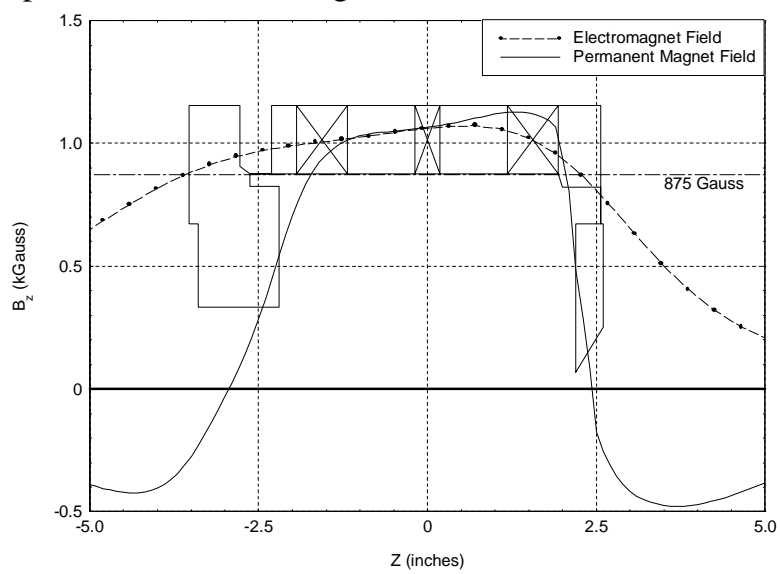

Figure 1. Comparison of magnetic fields of a PM solenoid and an "equivalent" electromagnet. The magnetic components of the PM-ECR source are illustrated by the thin outline. The permanent magnets are denoted by components with internal crossed lines.

The magnetic field profile of the PM-ECR source is comprised of three field "bumps" provided by three rings of permanent magnets. The lengths and strengths of these rings in the shell of the PM solenoid are variables in the baseline design that can be adjusted to tailor the solenoidal field profile. This baseline field profile can be further fine-tuned, without interrupting the operation of the ion source, by mechanically adjustable field shunts. Figure 2 shows the tuning range available in the baseline field of figure 1 when using these shunts. This combination of baseline field profile and field variability provided by the field shunts provides magnetic field versatility. In addition, substitution of various nonmagnetic (magnetic) parts with their corresponding magnetic (non-magnetic) equivalents provides another mechanism of coarse-tuning the magnetic field profile that is generally unavailable with electromagnet solenoids because of Stokes Theorem. 


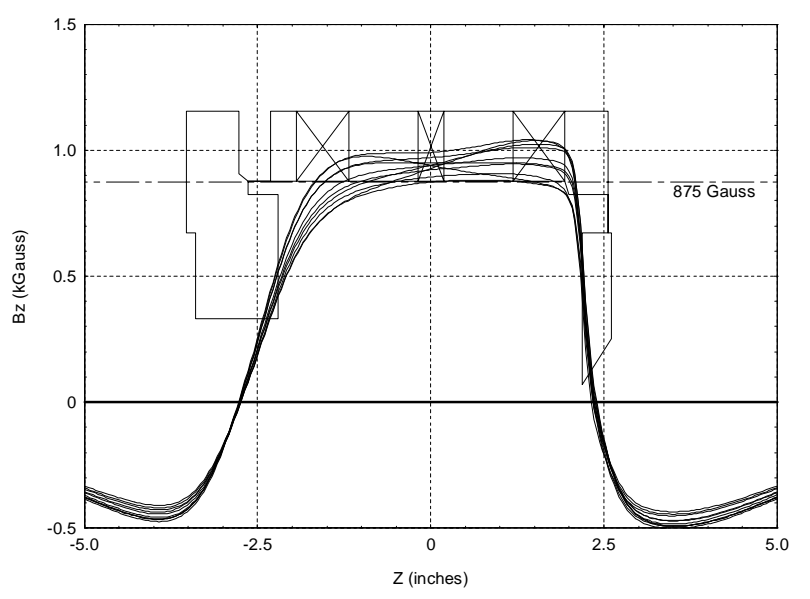

Figure 2. Illustration of the tuning range of the solenoidal magnetic field profile available via shunt tuners. The magnetic fields produced with the two shunts in a variety of different positions are shown by the collection of dark lines. As in figure 1, the permanent magnet shell and endcaps of the ion source are illustrated by the thin outline and the permanent magnet rings are denoted by the components with internal crossed lines.

\subsection{Mechanical Design Features}

The PM solenoid assembly is completely independent of the vacuum system. Hence the PM-ECR source can easily be adapted to mate with existing systems simply by substituting flanges. Also the ECR plasma chamber was designed to be removable from the ion source without affecting the high-voltage alignment or the permanent magnet solenoid. This design promotes easy replacement of ion source components 1) for maintenance, 2) to replace the plasma chamber to enable different ion beams that may require different plasma chamber characteristics, 3 ) to replace components that modify the magnetic field profile for different ions or ion beam requirements, and 4) to adjust the position or profile of the beam-formation aperture relative to the magnetic field profile to facilitate different ion beams or to optimize the beam characteristics for particular ion species.

The input waveguide can also be replaced easily by substituting either the waveguide end-flange of the ion source or by replacing an insert within that flange. In this manner the ion source can be coupled directly from a WR284 waveguide and $\mathrm{rf}$ window. Alternatively this PMECR ion source design supports alternative waveguide configurations that may prove beneficial to the performance and/or to the long-term reliability of the PMECR source.[2]

\subsection{Design Special Features}

In addition to the features of the baseline design discussed above, the following features are also available, either as a simple modification to the baseline design or by a adding or enabling optional features in that design.

The magnetic field profile shown in figures 1 and 2 illustrate the profile that has been shown to be efficient in the production of low charge-state ions.[3],[4] To facilitate the production of ions with a higher mean charge-state, the baseline magnetic field profile can be modified to produce the classical double-humped mirror field. Some of this adjust ment can be made through the addition of a third field-shunt. The third shunt reduces the strength of the center magnet and provides a magnetic mirror field configuration with small mirror ratios. Alternatively, the center magnet ring can be modified during fabrication to produce a mirror field configuration with mirror ratios of up to 2:1 (figure 3). In the former case, the fixed shunt can always be removed to reconfigure the magnetic field back into the low charge-state configuration. In the latter case, the mirror ratio becomes a permanent feature of the magnetic field. In either case, the two adjustable field shunts remain active to provide fine-tuning of the magnetic field during operation.

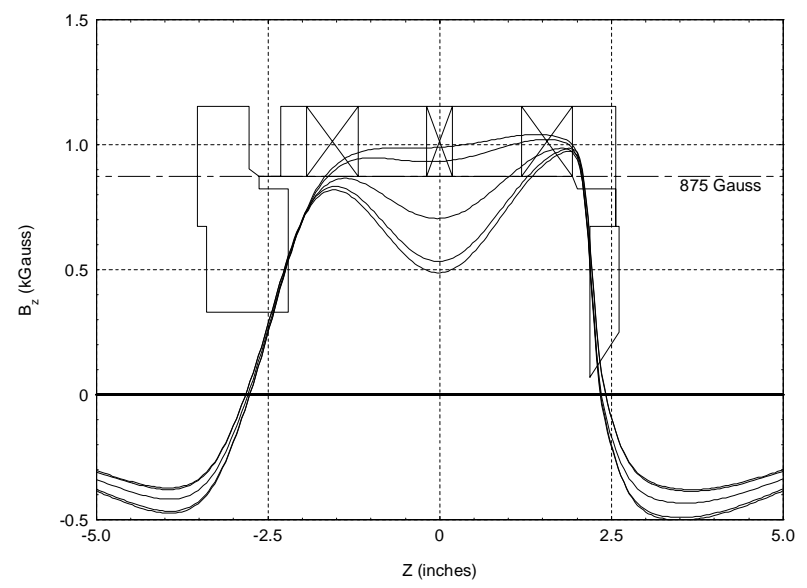

Figure 3. Field plot of a PM-ECR source showing the range of mirror field profiles available. As in figure 1, the permanent magnet shell and end-caps of the ion source are illustrated by the thin outline. The permanent magnet rings are denoted by the components with internal crossed lines.

\section{ECR ION SOURCE DESIGN DETAILS}

The high-current configuration of the PM-ECR source is illustrated in figure 4. This figure shows the ion source from the circular waveguide input end. Visible in the figure are the two shunt tuners with their screw-drive and drive belt assemblies, the waveguide endflange with its circular rf aperture, and the two end-flanges.

Fine-tuning the magnetic field profile is accomplished by sliding the magnetic shunts back and forth over the rings of permanent magnets using two sets 
of ball-screws. In the neutral position, these shunts have little effect on the magnetic field profile. In the maximum shunt position, a substantial portion of the magnetization of the permanent magnets is short-circuited and the local magnetic field magnitude is decreased.

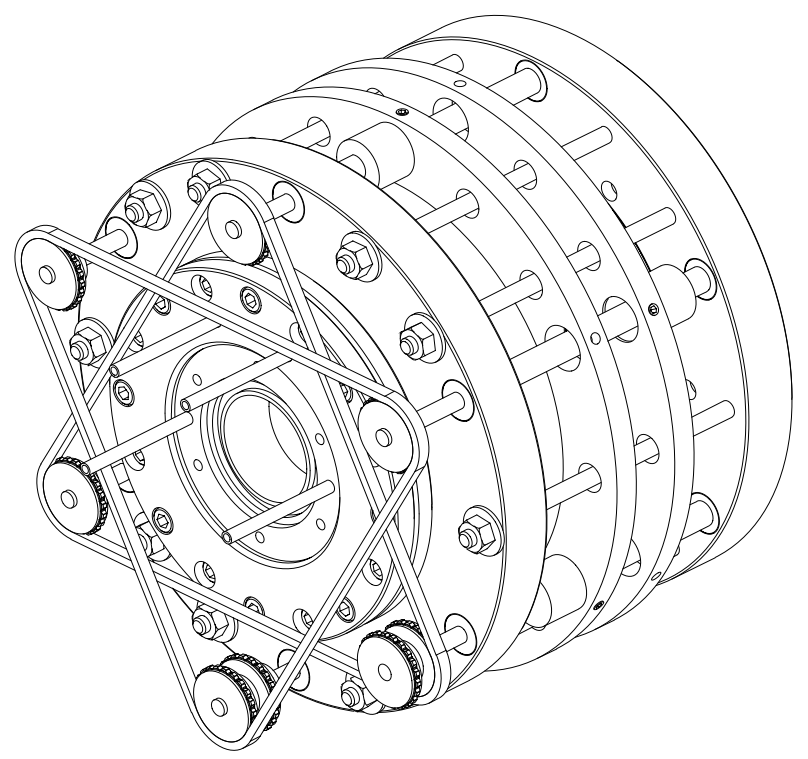

Figure 4. View of PM-ECR ion source assembly from the circular waveguide input end. The two magnetic field shunts are adjusted by rotating the three ball-screw supports for each ring via the two drive belts shown in the figure.

In the baseline design, the magnetic field magnitude increases monotonically from the rf window towards the beam aperture end. Alternate magnetic field configurations can be supplied with the more conventional mirror profiles (figure 3), however the simple ramp configuration of figure 1 has demonstrated superior performance in the production of low charge-state ions.[4],[5],[6]

The plasma chamber of the PM-ECR source was designed to be removable without disturbing the mounting or alignment of the ion source. This feature 1) facilitates maintenance, 2) enables different ion beams that may require different plasma chamber characteristics, 3) facilitates replacement of components that modify the magnetic field profile for different ions or ion beam requirements, and 4) allows adjustment of the position or profile of the beam-formation aperture relative to the magnetic field profile to facilitate different ion beams or to optimize the beam characteristics for particular ion species. All of these activities can be performed by simply swapping one plasma chamber insert for another without affect the alignment of the electrodes and can be performed with minimal interruption of service.

\section{CONCLUSION}

This PM-ECR ion source design has many unique features that promote flexibility in providing solutions to ion source problems. The permanent magnet assembly represents a significant improvement over electromagnet sources by eliminating many trouble-prone components. Specific features unique to PM solenoids provide magnetic field design flexibility unmatched by electromagnet systems. The baseline magnetic field is variable over a wide range of profiles. The shunt-tuners provide fine-tuning of magnetic fields to facilitate differing requirements of different ion beams and allow on-line fine tuning to accommodate long-term changes in ion beam characteristics due to plasma sputtering and aging or wearing of components.

The flexible mechanical design of the PM-ECR ion source provides adaptability to virtually any ion beam system simply by replacing the end-flanges. The plasma chamber can be removed and/or replaced easily 1) for maintenance, 2) to enable different ion beams that may require different plasma chamber characteristics, 3) to replace components that modify the magnetic field profile for different ions or ion beam requirements, and 4) to adjust the position or profile of the beam-formation aperture relative to the magnetic field profile to facilitate different ion beams or to optimize the beam characteristics for particular ion species.

\section{REFERENCES}

[1] J. D. Sherman, G. O. Bolme, L. D. Hansborough, D. J. Hodgkins, M. E. Light, E. A. Meyer, J. D. Schneider, H. V. Smith, Jr., M. W. Stettler, R. R. Stevens Jr., M. E. Thuot, T. J. Zaugg, and R. Ferdinand, in Proc. of the 1996 International Linac Conf, CERN 96-07, Geneva, Switzerland, (1996), p. 701

[2] W. D. Cornelius, "Waveguide Assembly and Circular Polarizer for $2450 \mathrm{MHz}$ ECR Ion Sources", these proceedings

[3] T. Taylor and J. F. Mouris, Nucl Instr \& Meth A336(1993)1

[4] T. Taylor and J. S. C. Wills, Nucl. Instr. \& Meth. A309(1991)37

[5] T. Taylor, Rev. Sci. Instr. 63(1992) 2507

[6] J. Sherman, D. Hodgkins, P. Lara, J. D. Schneider, and R. Stevens, Jr., in proc. 1995 Particle Accelerator Conference, Dallas TX, p 867 